\title{
ELEVEN NONEQUIVALENT CONDITIONS ON A COMMUTATIVE RING
}

\author{
ROBERT W. GILMER, JR.
}

\begin{abstract}
1. Introduction. We consider in this paper eleven conditions on a commutative ring $R$. The first of these is that $R$ contains an identity. It is well known that each of the other properties is a consequence of the first condition. This paper considers other relations which exist between these properties. A complete diagram of all simple implications which exist between the eleven properties, together with proof of these implications, is given in section 3. Examples illustrating simple implications which do not hold are presented in section 4. The notation and terminology is that of [10] with one exception: $\subseteq$ denotes containment and $\subset$ denotes proper containment. All rings considered will be assumed to be commutative and nonzero.
\end{abstract}

The eleven conditions considered on $R$, a commutative ring are:

$A: R$ contains an identity.

$B: R$ is generated by idempotent elements.

$C$ : If $A$ is a nonzero ideal of $R$ such that $\sqrt{A} \neq R$, then $R / A$ has an identity.

$D:$ If $x \in R$, there exists $y \in R$ such that $x=x y$.

$E:$ If $A$ is a proper ideal of $R, \sqrt{A} \neq R$.

$F: R$ is idempotent.

$G$ : Maximal ideals of $R$ are prime.

$H$ : If $P$ is a nonzero prime ideal of $R, R / P$ contains an identity.

$J$ : An ideal $A$ such that $\sqrt{A}$ is maximal is primary.

$K$ : Each proper ideal of $R$ is contained in a maximal ideal.

$L$ : If $A$ and $B$ are comaximal proper ideals of $R$, then $A B=A \cap B$.

It is well-known that properties $B-L$ follow from $A$. Further, each of these properties is preserved under homomorphisms. Rings satisfying $E$ have arisen naturally in [2], [3], and [4], and according to the terminology used

Received November 17, 1964. 
there, will be called $u$-rings. We shall need some preliminary results before establishing other relationships between these eleven properties.

2. Preliminary Results. One of our most frequent tools in this paper will be the passage from a commutative ring $R$ to $R^{*}$, where $R^{*}$ is obtained by adjoining an identity to $R$. [7; 83].

Theorem 1. Suppose $S$ is a commutative ring with identity $e$ and $R$ is a subring of $S$ such that $S=R[e]$. Then

(a) a subset $A$ of $R$ is an ideal of $R$ if and only if it is an ideal of $S$.

if $A$ is an ideal of $S$ and if $A \cap R$ is a finitely generated ideal of $R$, then $A$ is $a$. finitely generated ideal of $S$. (c) $R$ is Noetherian if and only if $S$ is Noetherian.

Proof. (a) is immediate.

To prove (b), let $a_{1}, \ldots, a_{k}$ be a basis for the ideal $A \cap R$. Then let $G$ be the set of integers $m$ such that $v+m e \in A$ for some $v \in R$. $G$ is a subgroup of the additive group of integers and is therefore cyclic, generated by some integer $q$. Let $v \in R$ be such that $v+q e=a \in A$. We shcw that $\left\{a_{1}, \ldots, a_{k}, a\right\}$ generates $A$ in $S$. Thus if $a^{\prime}=u+s e \in A$, then $s=m q \in G$. Hence $a^{\prime}-m a$ $=u-m v \in A \cap R$ so that $a^{\prime}-m a=\sum r_{i} a_{i}$ for some $r_{i} \in R$. It follows that $\left\{a_{1}, \ldots, a_{k}, a\right\}$ generates $A$ in $R$.

(c) : apply (a) and (b).

Theorem 2. Suppose $A, B, C$ are ideals of a ring $R$ such that $A$ has a basis $\left\{a_{1}, \ldots, a_{k}\right\}$ of $k$ elements and $A B=A C$. Then given $b \in B^{k}$, there is an element $c \in C$ such that $a b=a c$ for all $a \in A$. In particular, if $A$ contains a regular element $B^{k} \subseteq C$.

Proof. Let $R^{*}$ be a ring obtained by adjoining an identity to $R$. Theorem 1 shows that the hypotheses of Theorem 2 still hold if " $R$ " is replaced by " $R$ *". Hence we may assume $R$ contains an identity.

We first note that since $\left\{a_{1}, \ldots, a_{k}\right\}$ generates $A$ and since $B^{k}$ is generated by all products $v=b_{1} b_{2} \cdots b_{k}$ where $b_{i} \in B$, it suffices to prove the existence of a $c$ such that $a_{i} c=v a_{i}$ for $i=1,2, \ldots, k$. This we proceed to do.

We have $B A=\sum B a_{j}=C A=\sum C a_{j}$. Thus if $1 \leq i \leq k, b_{i} a_{i}=\sum_{j=1}^{k} c_{i j} a_{j}$, for some $c_{i j} \in C$. We therefore obtain a homogeneous system

$$
\text { (*) } \quad \sum\left(\delta_{i j} b_{i}-c_{i j}\right) a_{j}=0
$$


of $k$ equations linear in $a_{1}, \ldots, a_{k}$ and having coefficients in $R$. If $d$ is the determinant of the system $(*), a_{i} d=0$. for $i=1,2, \ldots, k$. It is easily seen, however, that $d=v-c$ for some $c \in C$. Theorem 2 now follows.

Corollary $1^{11}$. Let $A$ and $B$ be ideals of a ring $R$ such that $A$ is finitely generated and such that $A B=A$. Then there exists $b \in B$ such that $a b=a$ for all $a \in A$.

Proof. As in the proof of Theorem 2, we may assume $R$ has an identity element $e$, so that $A B=A R$. Suppose $A$ is generated by $k$ elements. Then by Theorem 2, given $e \in R^{k}$, there exists $b \in B$ such that for all $a \in A, a b=a e$ $=a$.

Corollary $2^{21}$. A finitely generated idempotent ideal $B$ of a ring $R$ is principal and is generated by an idempotent element.

Proof. Apply Corollary 1.

Corollary 3. Suppose $\left\{x_{1}, \ldots, x_{n}, y_{1}, \ldots, y_{n}\right\}$ is a collection of elements of a ring $R$ such that $x_{i} y_{i}=x_{i}$ for each $i$. There exists an element $y \in R$ such that $x_{i} y=x_{i}$ for each $i$.

Proof. Let $A$ be the ideal of $R$ generated by $\left\{x_{1}, \ldots, x_{n}\right\}$ and $B$ the ideal of $R$ generated by $\left\{y_{1}, \ldots, y_{n}\right\}$. The hypothesis on $x_{i}$ and $y_{i}$ imply $A B=A$. Corollary 3 now follows from Corollary 1.

3. Relations Between the Eleven Properties. The following diagram describes completely the simple implications which exist between these properties. In particular, no two of the eleven properties are equivalent.

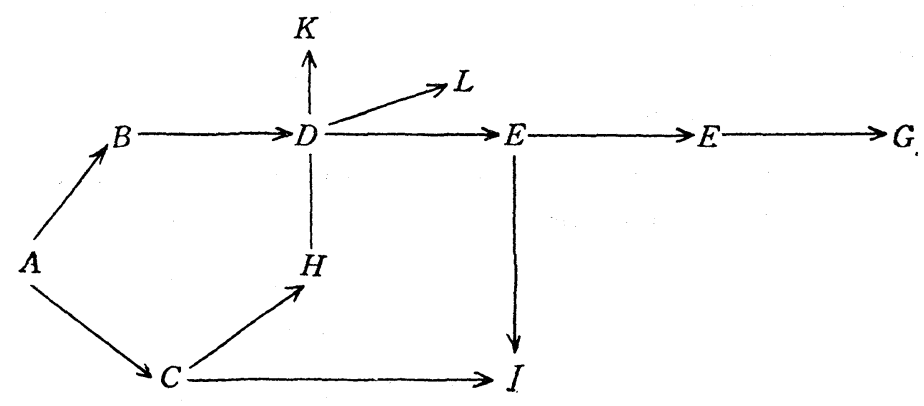

1) Corollary 1 is proved in [10;215], for rings with identity.

2) Corollary 2 was first proved by Mori in $[8 ; 174-175]$. 
Before proving that these twelve implications hold, we first give equivalent statements of properties $C, D, E$, and $H$.

Proposition 1. $C$ is equivalent to $C^{\prime}$;

$C^{\prime}$ : If $A$ is a nonzero principal ideal of $R$ such that $\sqrt{A} \subset R$, then $R / A$ has an identity.

Proposition 2. $D$ is equivalent to each of $D^{\prime}, D^{\prime \prime}, D^{\prime \prime \prime}$ :

$D^{\prime}$ : For each ideal $A$ of $R, R A=A$.

$D^{\prime \prime}$ : If $\left\{x_{1}, \ldots, x_{u}\right\}$ is a finite set of elements of $R$, there exists $y \in R$ such that $x_{i} y=x_{i}$ for each $i$.

$D^{\prime \prime \prime}$ : If $A$ and $B$ are comaximal ideals of $R$, then $A \cap B=A B$.

Proof. That $D$ implies $D^{\prime \prime}$ follows from Corollary 3 . It is clear that $D^{\prime \prime}$ implies $D^{\prime}$ and $D^{\prime}$ implies $D^{\prime \prime \prime}$ by the usual proof. $[9 ; 40]$.

If $D^{\prime \prime \prime}$ holds in $R$ and $x \in R$, then $(x)$ and $R$ are comaximal so that $(x)=$ $R \cap(x)=R x$. Hence $x \in R x$ and $D$ holds.

Proposition 3. $E$ is equivalent to $E^{\prime}$ :

$E^{\prime}:$ Each proper ideal of $R$ is contained in a proper prime ideal.

Proof. This follows immediately from the fact that the radical of an ideal is the intersection of the prime ideals containing it. $[7 ; 104]$.

Proposition 4. $H$ is equivalent to $H^{\prime}$ :

$H^{\prime}$ : If $Q$ is a nonzero primary ideal with radical $P \neq R, R / Q$ contains an identity element.

Proof. See Lemma 3 of [2].

We now prove the implications of our chart. That $A \rightarrow B, A \rightarrow C, E \rightarrow F$, and $C \rightarrow H$ are immediate.

That $B \rightarrow D$ follows immediately from Corollary 3 .

Note that a domain in which $D$ holds has an identity. Hence $D \rightarrow H$.

That $D \rightarrow L$ follows from Proposition 2.

$D \rightarrow E$ : Suppose $A \subset R$ and $x \in R-A$. If $y$ is such that $x=x y$, then for any $n, x=x y^{n}$. Hence $y^{n} \notin A$ and $\sqrt{A} \subset R$ so that $E$ holds.

Note that the ideal $M$ of $R$ is maximal if and only if $R / M$ is a ring having exactly two ideals. By $[10 ; 133]$, the ring $S$ has exactly two ideals if and only if $S$ is a field or the additive group of $S$ is cyclic of prime order and 
multiplication in $S$ is trivial (the product of any two elements in zero). Hence if $M$ is a maximal ideal of $R, M$ is not prime if and only if $M \supseteq R^{2}$. Equivalently, $M$ is prime if and only if $\sqrt{M} \neq R$. From these observations it is clear that $F \rightarrow G$.

The following lemma shows that $C \rightarrow J$.

Lemma 1. Let $A$ be an ideal of a ring $R$ such that $\sqrt{A}$ is maximal in $R$. Then $A$ is primary if and only if $R / A$ has an identity element.

Proof. If $R_{\prime}^{\prime} A$ has an identity, then $A / A$ is primary in $R / A$ since $\sqrt{A} / \bar{A}$ $=\sqrt{A / A}$ is maximal. Hence $A$ is primary in $R[10 ; 148-153]$.

Conversely, if $A$ is primary, then $\sqrt{A}$ is prime and maximal. Our previous observations show $R / \sqrt{A}$ is a field. Lemma 3 of [2] now shows $R / A$ has an identity.

$D \rightarrow K$ : If $W \subset R, W$ is contained in a proper prime ideal $P$ since $D \rightarrow E \leftrightarrow E^{\prime}$. But $D$ also implies $H$ so that $R / P$ contains an identity. Thus $P / P$ is contained in $M / P$, a maximal ideal of $R / P$. Then $A \subseteq P \subseteq M$ and $M$ is maximal in $R$ (Note that we have actually shown $E$ and $H$ imply $K$. Theorem 3 shows $E$ and $H$ are equivalent to $D$.).

We delay for the present the proof that $E \rightarrow J$.

4. Examples. The following series of examples shows that the only simple implications which exist between our eleven properties are those shown in the diagram in section 3. It can be checked that these examples are sufficient to show no other simple implication exists, though we shall not enumerate those illustrated by most examples.

EXAmple 1. Let $Q$ be a nonzero additive abelian group. $Q$ becomes a ring $R$ if multiplication is defined trivially. For any such $Q, F$ does not hold in $R$, while $C$ holds vacuously.

If $Q$ is cyclic of order $12, K$ holds in $R$ while $G$ and $L$ do not.

If $Q$ is cyclic of order $6, K$ and $L$ hold in $R$ but $G$ does not.

Finally, if $Q$ is the additive group of rationals, then $G$ holds in $R$ while $L$ and $K$ do not.

These examples show, for instance, that $H$ and $J$ are the only properties implied by $C$.

Example 2. We let $R$ be the ring of all finite subsets of $Z$. $B$ holds in 
$R$ but $C$ does not.

Example 3. We let $R$ be the ring of even integers. $R$ is a one-dimensional Noetherian domain in which $K$ and $H$ hold, but $G, J$, and $L$ do not. $G$ fails because (4) is maximal but not prime. (12) has radical (6), a maximal ideal, but (12) is not primary in $R$ so that $J$ fails also. $L$ fails because (4) and (6) are comaximal but $(4) \cap(6)=(12) \supset(24)=(4)(6)$.

ExAmpLe 4. Let $Q$ be a valuation ring whose maximal ideal $M$ is the union of the prime ideals properly contained in $M$ and such that $Q=k+M$ where $k=G F(p)$ for some prime $p$ (for example, see $[5$, section 5$]$ ). If $W$ is an ideal of $Q$ such that $W \subset M$, then given $m \in M-W, m$ is in some prime ideal $P \subset M$ so that $W \subseteq(m) \subseteq P \subset M$ and so $\sqrt{W} \subseteq P \subset M$. Now consider $R=M$. Because $Q=k+M, R$ and $Q$ have exactly the same ideals $(\neq R)$ by Theorem 1. Hence the ideals of $R$ are linearly ordered and the observation just made shows that $R$ is a $u$-domain. Also, $L$ holds vacuously in $R$. We have already shown $K$ fails in $R$ and a subsequent result (Theorem 3 ) shows that $H$ also fails in $R$, for clearly $R$ does not have an identity.

EXAMPLE 5. Let $k$ be a field and let $\left\{X_{i}\right\}_{i=1}^{\infty}$ be a set of elements from an extension field of $k$ which are algebraically independent over $k$. Let $M$ be the ideal of $Q=k\left[\left\{X_{i}\right\}\right]$ generated by $\left\{X_{i}\right\}$ and let $N$ be the ideal of $Q$ generated by $\left\{X_{i}-X_{i} X_{j}\right\}_{i<j}$. Then define $R=M_{/} / N$. That $D$ holds in $R$ is shown by Corollary 3. In [1] it is shown that $R$ contains no idempotent element, and hence $B$ fails in $R$. Theorem 3 then implies $C$ does not hold in $R$.

Example 6. Suppose $R$ is the principal ideal generated by $X$ in the polynomial domain $S=Z[X]$. Since $S=Z+R, R$ is a Noetherian domain by Corollary 3. Hence $K$ holds in $R$. If $V$ is the ideal of $S$ generated by $2 X$ and $X+X^{2}$ and if $W$ is the ideal of $S$ generated by $3 X$ and $X^{2}, V$ and $W$ are comaximal maximal ideals of $R, W$ is not prime, and $6 X \in V \cap W-(V W)$. Hence $G$ and $L$ fail in $R$. Also $2 X S+\left(X^{2}+X^{4}\right) S$ has radical $V$ but is not primary in $R$ so that $J$ does not hold in $R$. Finally $\left[2 X+X^{2}\right] S$ is prime in $R$ but $R /\left[2 X+X^{2}\right] S$ does not have an identity so that $H$ is invalid in $R$.

EXAMPLE 7. For $k$ a positive integer, let $\theta_{k}=2^{2^{k}} \sqrt{2}$ and let $S_{k}=Z\left[\theta_{k}\right] . \quad S_{k}$ is the integral closure of $Z$ in $\Gamma\left(\theta_{k}\right), \Gamma$ the field of rationals. Let $T_{k}=\left\{2 a_{0}+\right.$ $\left.a_{1} \theta_{k}+\cdots+a_{2^{k-1}} \theta_{k}^{2^{k}-1} \mid a_{i} \in Z\right\}$. Because $S_{k}$ is integral over $Z$, there is a maximal 
ideal $M_{k}$ of $S_{k}$ such that $M_{k} \cap Z=(3)$. If $E$ denotes the ring of even integers, we have $M_{k} \cap E=(6)$. Hence $P_{k}=M_{k} \cap T_{k}$ is a maximal ideal of $T_{k}$ ( $S_{k}$ is integral over $T_{k}$ ) such that $P_{k} \cap E=(6)$.

Next we note that $T_{k}^{2^{k+1}} \cap E=(4)$. For $4=\theta_{k}^{2^{k+1}} \in T_{k}^{2^{k+1}}$ and if $d_{1}, \ldots, d_{2^{k+1}}$ $\in T_{k}$, then for each $i, b_{k}=d_{i} / \theta_{k} \in T_{k}$. Therefore $d_{1} d_{2} \cdots d_{2^{k+1}}=4 b_{1} \cdots b_{2^{k+1}}$ so that $E \cap T_{k}^{2^{k+1}}=(4)$ as asserted. Consequently, if $W_{k}=P_{k} \cap T_{k}^{2^{k+1}}, W_{k}$ has maximal radical $P_{k}$ in $T_{k}$ and $W_{k} \cap E=(12)$.

We observe that having chosen $M_{k}$, we may choose $M_{k+1}$ in $S_{k+1}$ so that $M_{k+1} \cap S_{k}=M_{k}$ because $S_{k+1}$ is integral over $S_{k}$. Since also $T_{k} \subseteq T_{k+1}^{2}, T_{k}^{2^{k+1}} \subseteq$ $T_{k+1}^{2^{k+2}}$. It follows then that $W_{k} \subseteq W_{k+1}$. Now let $T=\cup T_{k}, P=\cup P_{k}, W=\cup W_{k}$. The following facts concerning $T$ are easily checked: $T$ is an idempotent onedimensional domain, $P$ is maximal in $T$ and $P \cap E=(6)$, and $\sqrt{ } W=P, W \cap E$ =(12). Consequently, $W$ is not primary in $T ; 2 \cdot 6 \in W, 6 \notin W$, and $2 \notin P$. Hence $F$ holds in $T$ but $J$ does not.

Example 8. Let $S=Z\left[X_{1}, X_{2}, \ldots,\left\{U_{i j}\right\}\right]$ be a polynomial domain over $Z$, let $M$ be the ideal of $S$ generated by $\left\{2 X_{1}, 2 X_{2}, \ldots\right\}$ and let $N$ be the ideal of $S$ generated by $\left\{2 X_{i}-2^{i+1} U_{i j} X_{i+1}^{j+1}\right\}$ for all positive integers $i$ and $j$. Let $R=M / N$. Let $x_{i}$ be the $N$-residue of $x_{i}, u_{i j}$ the $N$-residue of $U_{i j}$. We have, for any $i$ and $j, 2 x_{i}=\left(2 u_{i j} x_{i+1}\right)\left(2 x_{i+1}\right)^{j}$. Hence if $W$ is an ideal of $R$ having radical $R$, then for any $i, W$ contains some power of $2 x_{i+1}$ and therefore contains $2 x_{i}$. Therefore $W=R$ and $R$ is a $u$-ring. Yet $L$ does not hold in $R$. If $T$ is the ideal of $S$ generated by $\left\{6 x_{1}, 6 x_{2}, \ldots\right\}$ and if $V$ is the ideal of $S$ generated by $\left\{10 x_{1}, 10 x_{2}, \ldots\right\}$, then $A+T / A$ and $A+V / A$ are comaximal ideals of $R$ whose intersection properly contains their product.

Example 9. If $R=2 Z / 12 Z, L$ holds in $R$ but $J$ does not.

5. Other Relations between the Eleven Properties. The examples of the previous section serve to point out that properties $C$ and $H$ may be satisfied vacuously in a ring and are therefore not generally strong properties. In this section we show that $C$ and $H$ are rather strong properties in a $u$-ring. In fact we show that if $C$ holds in an idempotent ring $R$, then $R$ has an identity and we show that $E$ and $G$ are equivalent to $D$. We also investigate relations between our eleven properties in the case when $R$ is Noetherian or, less restrictively, finitely generated. We first consider a lemma. 
Lemma 2. Let $V$ be a proper ideal of a commutative ring $R$ and let $P$ be $a$ minimal prime ideal of $V$ such that $R / P$ has an identity $\bar{e} \neq \overline{0}$. If $W=$ $\{x \in R \mid t x-x \in V$ for some $t \in R\}$, then $W$ is an ideal of $R$ and $V \subseteq W \nsubseteq P$.

Proof. If $e_{i} x_{i}-x_{i} \in V$ for $i=1,2$ and if $t=e_{1}+e_{2}-e_{1} e_{2}$ then $t x_{1}-x_{1}=$ $\left(e_{1} x_{1}-x_{1}\right)-e_{2}\left(e_{1} x_{1}-x_{1}\right) \in V$ and similarly $t x_{2}-x_{2} \in V$ so that $t\left(x_{1}-x_{2}\right)-\left(x_{1}-x_{2}\right) \in V$ and $x_{1}-x_{2} \in W$. Further, if $r \in R$, then $e_{1} r x_{1}-r x_{1}=r\left(e x_{1}-x_{1}\right) \in V$ so that $r x_{1} \in A$. Hence $W$ is an ideal. Obviously $V \subseteq W$.

Now if $Q$ is the isolated primary component of $V$ belonging to $P, R / Q$ has an identity $\bar{s}$. Thus if $y \in R, s y-y \in Q$ so that $(s y-y) v_{y}=s\left(y v_{y}\right)-\left(y v_{y}\right) \in V$ for some $v_{y} \notin P$. This implies $y v_{y} \in W$. In particular, if $y \notin P$, then $y v_{y} \in W-P$.

Theorem 3. $D \leftrightarrow E$ and $H$.

Proof. By the results of section 3, we need only show that if $H$ holds in the $u$-ring $R$, then so does $D$.

Since $R=R^{2} \neq(0)$, there is an element $x \in R$ such that $R x \neq(0)$. We let $W_{1}=\{y \in R \mid y t-y \in R x$ for some $t \in R\}$ and we let $W_{2}=\{z \in R \mid z s-z \in W$, for some $s \in R\}$. Now $W_{1} \subseteq W_{2}$ by Lemma 2. But if $z \in W_{2}$ and $z s-z \in W_{1}$, then $(z s-z) t-(z s-z)=(s t-t-s) z-z \in R x$ so that $z \in W_{1}$. Hence $W_{1}=W_{2}$. But $(0) \subset R x \subseteq W_{1}$ so any minimal prime of $W_{1}$ is nonzero. Lemma 2 then implies that $R$ is a minimal prime of $W_{1}$ so that $W_{1}=R$ since $R$ is a $u$-ring. In particular, then, $x \in W_{1}$ so that $t x-x \in R x$ and $x \in R x$.

Now let $V_{1}=\{v \in R \mid t v=v$ for some $t \in R\}$. As just shown, $(0) \subset(x) \subseteq V_{1}$. Let $V_{2}=\left\{w \in R \mid s w-w \in V_{1}\right.$ for some $\left.s \in R\right\}$. A repetition of the argument in the preceding paragraph shows $V_{1}=V_{2}=R$ so that $D$ holds in $R$.

COROLlary 4. Suppose the ascending chain condition for prime ideals holds in the $u$-ring $R$. Then $D$ holds in $R$.

Proof. We show that $H$ holds in $R$. Thus let $P$ be prime in $R$ and maximal with respect to the property that $R / P$ does not have an identity. Then $R / P$ is a $u$-domain in which $H$ holds. By Theorem $3, D$ holds in $R / P$. As previously observed, this implies $R / P$ contains an identity. Hence there is no prime ideal $P$ of $R$ such that $A$ does not hold in $R / P$. That is, $H$ holds in $R$. Corollary 4 then follows from Theorem 3.

Proposition 5. Suppose $W$ is an ideal of a u-ring $R$ such that $\sqrt{W}=M, a$ 
maximal ideal. If $x, y \in R$ are such that $x y \in W$ and $y \notin M$, then $R x \subseteq W$.

Proof. Since $y \notin M, M+(y)=R$. Hence, if $q \in R$, there exists $m, v \in R$, $\alpha \in Z$ such that $q=m+v y+\alpha y$. For some integer $t, m^{t} \in W$. Hence $q^{t}=$ $m^{t}+v^{\prime} y+\alpha^{\prime} y$ and $x q^{t}=m^{t} x+v^{\prime} x y+\alpha^{\prime} x y \in W$. This shows that $\sqrt{W: x}=R$. Since $R$ is a $u$-ring, $R=W: x$ so that $R x \subseteq W$ as asserted.

THEOREM 4. $J$ holds in the u-ring $R$.

Proof. Suppose the ideal $W$ of $R$ has maximal radical $M$, that $x y \in W$ and $y \notin M$. By proposition 5, $R x \subseteq W$. Now $R / W$ is a $u$-ring in which $H$ holds since $M / W$ is the only proper prime ideal of $R / W$. By Theorem $3, D$ holds in $R / W$. Whence $\bar{x} \in \bar{x}(R / W)=x R+W / W=W / W$ so that $x \in W$. Hence $W$ is primary.

TheOREM 5. An idempotent ring $R$ in which $C$ holds contains an identity element.

Proof. Since $R=R^{2} \neq(0)$, there exists $x \in R$ such that $R x \neq 0$. Let $W_{1}=$ $\{y \in R \mid y t-y \in R x$ for some $t \in R\}$. Now $(0) \subset R x \subseteq W_{1}$ so that $R / W_{1}$ has an identity $\bar{e}$. Then $e x-x \in R x$ and $x \in R x$. This shows that if $W_{2}=\{y \in R \mid y=y t$ for some $t \in R\}$, then $(0) \subset(x) \subseteq W_{2}$ so that $R / W_{2}$ has an identity $\bar{u}$. Thus if $r \in R, r u-r \in W_{2}$ and hence $r u-r=(r u-r) t$ for some $t \in R$. It follows that $r=(u+t-u r) r$ so that $r \in W_{2}$-that is, $W_{2}=R$ and $D$ holds in $R$.

We suppose $R$ does not contain an identity. Then $R$ contains no regular element. Then let $x \in R$ and let $y$ be such that $x=x y$. Since $y$ is a zero divisor, $y z=0$ for some $z \neq 0$. We note that $(x) \cap(z)=(0)$ for if $r x=s z$, then $r x y=r x$ $=s z y=0$. Finally, $R /(x)$ and $R /(z)$ have identities $\overline{e_{1}}, \overline{e_{2}}$ respectively-that is $e_{1} t-t \in(x)$ and $e_{2} t-t \in(z)$ for all $t \in R$. Now if $e=e_{1}+e_{2}-e_{1} e_{2}$, then for all $t \in R$, et $-t=e_{1} t-t-e_{2}\left(e_{1} t-t\right)=e_{2} t-t-e_{1}\left(e_{:} t-t\right) \in(x) \cap(z)=(0)$. Hence $e$ is an identity of $R$, contrary to our assumption. Therefore $R$ has an identity.

EXAmpLe 10. Let $Q$ be a nondiscrete rank one valuation ring with maximal ideal $M$ such that $Q=\pi+M$ for some field $\pi=G F(p)$. Consider $R=M . \quad R$ is an idempotent ring and $H$ holds vacuously in $R$. But clearly $D$ does not hold in $R$. Hence $H$ and $F$ do not imply $D$.

We now consider the case of a finitely generated ring $R$. In any such ring $K$ holds, for if $\left\{P_{\alpha}\right\}$ is a chain of proper ideals of $R$, then $\cup P_{\alpha}$ is also a proper ideal. 
THEOREM 6. If $G$ holds in the finitely generated ring $R$, then $R$ has an identity.

Proof. If $W$ is a proper ideal of $R, W$ is contained in a maximal ideal $M$ since $K$ holds in $R$. Since $G$ holds, $M$ is prime so that $R$ is a $u$-ring by Proposition 3 . In particular, $R$ is finitely generated and idempotent so that Corollary 2 shows that $R$ contains an identity.

In the course of the proof of Theorem 6 we have observed that $K$ and $G$ imply $E$. Examples 1, 3, 6, and 9 show that if simple implications other than those of the form $X \rightarrow K$ and those given in section 3 exist under the additional hypothesis that $R$ is Noetherian, then these other implications are among $J \rightarrow H$ and $L \rightarrow H$. We shall see at once that the first of these two implications does not hold.

ExAmple 11. Let $S=Z[X]$ be a polynomial domain over $Z$, let $M=(2, X)$ and let $T=S_{M}$. We note that if $\alpha \in T-M T$, then $\alpha-1 \in M T$ so that $T=$ $Z+M T$. Hence $M T$ is a Noetherian ring. We let $R=M T / X^{2} T$. Then $R$ is also Noetherian. $X T / X^{2} T$ is the only proper prime ideal of $R$, and it is easily checked that $M T / X T$ is not idempotent, and thus has no identity. Therefore $H$ does not hold in $R$. But $J$ holds vacuously; no ideal of $R$ has maximal radical.

The next example shows that $H$ need not hold in a finitely generated ring $R$ in which $J$ and $L$ hold.

ExAmple 12. Let $S$ be a rank two valuation ring whose maximal ideal $M$ is principal and $S$ is such that $S=\pi+M$ where $\pi=G F(p)$ for some prime $p$ (such an $S$ may be constructed in $\pi(X, Y, Z)$ ). Let $R=M$. As in example 10, $J$ holds vacuously. Because the ideals of $R$ are linearly ordered, $L$ is also vacuously satisfied. $R$ is finitely generated because $M$ is principal and $S=$ $\pi+M$. Yet $H$ does not hold in $R$. If $P$ is the nonzero prime ideal of $S$ properly contained in $M,[M / P]^{2}=M^{2}+P / P=M^{2} / P \subset M / P$ so that $M / P$ does not have an identity.

We are unable to determine whether $H$ holds in a Noetherian ring satisfying $L$. The best result obtained in this direction is Theorem 7. The proof uses the following lemma. 
Lemma 3. Suppose $L$ holds in the finitely generated domain $Q$ and that $Q$ does not contain an identity. Then $Q$ is principal. If $Q$ is Noetherian, if $K$ is the quotient field of $Q$, and if $Q^{*}=Q[e]$ is the subring of $K$ generated by $Q$ and the identity element $e, Q^{*}$ is a one-dimensional local domain and each ideal of $Q^{*}$ contains a power of $Q$.

Proof. Theorem 6 implies there is a maximal ideal $M$ of $Q$ such that $M$ is not prime. Hence $Q^{2} \subseteq M$ so that if $x, y \in Q-M$, then $M+(x)=Q$, but $x y \in M \cap(x), x y \notin M(x)$. Since $L$ holds in $Q$, we must have $Q=(x)$.

Suppose now that $Q$ is Noetherian and $Q^{*}$ is not a local domain. Then there exist distinct maximal ideals $M_{1}, M_{2}$ and elements $m_{i} \in M_{i}$ such that $m_{1}+m_{2}=1$. Then $m_{1} Q$ and $m_{2} Q$ are proper comaximal ideals of $Q$. Hence $m_{1} Q m_{2} Q=m_{1} Q \cap m_{2} Q=\left[\left(m_{1}\right) \cap\left(m_{2}\right)\right] Q$, since $Q$ is principal, $=m_{1} m_{2} Q$. But $Q^{*}$ is a domain, and hence $e \in Q$. This contradiction shows that $Q^{*}$ is a local domain. Hence $Q^{*} / Q$ is also local. Since $Q^{*} / Q$ is a homomorphic image of $Z, Q^{*} / Q \simeq Z /\left(p^{k}\right)$ for some prime $p$ and some integer $k \geq 1$. Hence $Q$ is primary for the maximal ideal $M$ of $Q^{*}$; in fact $M=Q+(p e)$ and $p^{k} e \in Q$. Because $Q$ is principal, the principal ideal theorem $[10 ; 238]$ shows $M$ is a minimal prime of $Q^{*}$. Consequently, $Q^{*}$ is one-dimensional. That each ideal of $Q^{*}$ contains a power of $Q$ now follows simply because $Q^{*}$ is Noetherian.

THEOREM 7. If $L$ holds in the finitely generated ring $R$, then $R$ is principal or $H$ holds in $R$. If $R$ is Noetherian and $P$ is a proper prime ideal of $R$ which is not both a maximal prime and a minimal prime of $R$, then $R / P$ contains an identity.

Proof. Suppose $R$ is finitely generated and $H$ does not hold in $R$. Let $P$ be a prime ideal of $R$ such that $R / P$ does not have an identity. By Lemma 3, $R / P$ is principal. $R=P+(x)$ for some $x$. Then also $R=\left[P+\left(x^{2}\right)\right]+(x)$ and because $R / P$ does not have an identity $x \notin P+\left(x^{2}\right)$ and $x^{2} \notin P+\left(x^{3}\right) \supseteq P(x)+\left(x^{3}\right)$. Since $x^{2} \in\left[P+\left(x^{2}\right)\right] \cap(x)$ and $L$ holds in $R, R=(x)$.

To prove the last assertion, it suffices to show that if $P_{1}$ and $P_{2}$ are prime in the Noetherian ring $R$ satisfying $L$ with $P_{1} \subset P_{2} \subset R$, then $R / P_{1}$ has an identity. But this follows immediately from Lemma 3 ; since $P_{2} / P_{1}$ contains no power of $R / P_{1}, R / P_{1}$ has an identity. 


\section{REFERENCES}

[1] R. Gilmer, A courterexample to a conjecture of Krull, submitted for publication.

[2] R. Gilmer, Extension of results concerning rings in which semiprimary ideals are primary, Duke Math. J., 31 (1964), pp. 73-78.

[ 3 ] R. Gilmer, Rings in which semi-primary ideals are primary, Pac. J. Math., 12 (1962), pp. 1273-1276.

[4] R. Gilmer and J. Mott, Multiplication rings as rings in which ideals with prime radical are primary, to appear in Trans. Amer. Math. Soc.

[5] R. Gilmer and J. Ohm, Primary ideals and valuation ideals, to appear in Trans. Amer. Math. Soc.

[6] W. Krull, Idealtheorie, New York (1948).

[7] N. McCoy, Rings and Ideals, Menasha, Wisconsin (1948).

[ 8 ] S. Mori, Über Ringe in denen die grossten Primarkomponenten jedes Ideals eindeutig bestimmt ist, Hiroshima Journal 1 (1931), pp. 159-193.

[9] B. L. van der Waerden, Modern Algebra, V. II, New York (1950).

[10] O. Zariski and P. Samuel, Commutative algebra, V. I., Princeton (1958).

Florida State University

Tallahassee, Florida

Note: References [4] and [5] have now appeared. [1] is to appear in the American Mathematical Monthly.

[4] R. Gilmer and J. Mott, Multiplication rings as rings in which ideals with prime radical are primary, Trans. Amer. Math. Soc.. 114 (1965), pp. 40-52.

[5] R. Gilmer and J. Ohm, Primary ideals and Valuation ideals, Trans. Amer. Math. Soc., 117 (1965), pp. 237-250. 